\begin{tabular}{lcc} 
& BNL-63127 \\
\hline \hline May 1996 & RHIC DETECTOR NOTE & RHIC/DET Note 20
\end{tabular}

Correlated forward-backward Coulomb dissociation and luminosity monitoring at heavy ion colliders

\author{
A.J. Baltz and S.N. White \\ Brookhaven National Laboratory \\ Upton, N.Y. 11973
}

Under Contract No. DE-AC02-76CH00016 with the

UNITED STATES DEPARTMENT OF ENERGY 


\section{DISCLAIMER}

This report was prepared as an account of work sponsored by an agency of the United States Government. Neither the United States Government nor any agency thereof, nor any of their employees, makes any warranty, express or implied, or assumes any legal liability or responsibility for the accuracy, completeness, or usefulness of any information, apparatus, product, or process disclosed, or represents that its use would not infringe privately owned rights. Reference herein to any specific commercial product, process, or service by trade name, trademark, manufacturer, or otherwise does not necessarily constitute or imply its endorsement, recommendation, or favoring by the United States Government or any agency thereof. The views and opinions of authors expressed herein do not necessarily state or reflect those of the United States Government or any agency thereof. 



\title{
Correlated forward-backward Coulomb dissociation and luminosity monitoring at heavy ion colliders
}

\author{
A. J. Baltz and S. N. White \\ Brookhaven National Laboratory, Upton, New York 11973
}

(May 13, 1996)

\begin{abstract}
We present an expression for the rate of correlated Coulomb dissociation 'of both colliding nuclei at a heavy-ion collider. For the case of $\mathrm{Au}+\mathrm{Au}$ at RHIC, the cross section for mutual correlated dissociation is about 3 barns. This process should be cleanly measured with very forward (zero degree) calorimeters proposed for RHIC. We also discuss prospects for luminosity monioring using this process.

PACS: 34.90. $+q, 25.75+r$
\end{abstract}

\section{INTRODUCTION}

The heavy ion collider experiments now being built for RHIC and the LHC will require, for their physics programs, luminosity monitoring devices. These monitors record a relatively well understood process simultaneously with the measurements of primary interest thereby making it possible to normalize relative to the monitor cross section.

For example, at $\mathrm{e}^{+} \mathrm{e}^{-}$and $\mathrm{e}-\mathrm{p}$ colliders the luminosity monitoring device is a pair of calorimeters placed close to the beam direction which measure the energy and angle of outgoing particles from Bhabha scattering and Bethe-Heitler bremstrahlung ( e-p $\rightarrow$ e-p- $\gamma$ ) respectively.

At hadron colliders (ie $\bar{p}$ p) a luminosity monitor based on an arbitrary fraction of 
the inelastic hadronic cross section has been used. Under special running conditions it has also been possible to measure directly elastic pp or $\bar{p}$ p scattering with momentum transfers small enough so that the Coulomb contribution is dominant $\left(|t| \sim .001 \mathrm{GeV}^{2} / \mathrm{c}^{2}\right)$ ). This process can then be used to calibrate the monitor [3]. Another calibration procedure which has been used is the so-called "Luminosity independent method" for determining the total cross- section [6]. Since, at high energies the hadronic scattering amplitude is almost purely imaginary, an extrapolation of the differential elastic scattering rate to $|t|=0.0$ can, through the optical theorem, provide a measure of the total hadronic cross section. The alternate way of measuring the total cross section - summing up the scattering rate in all channels- depends on a different power of the luminosity ( linear instead of square root) so the luminosity calibration is obtained as a by-product.

In the case of heavy ion collisions, elastic scattering is essentially unmeasurable because of the small scattering angles involved but there are a number of large cross section QED processes which could be exploited for luminosity monitoring.

In this report we present calculated cross sections for mutual correlated Coulomb dissociation at RHIC and LHC. We argue that this reaction, because it yields correlated neutrons in each beam direction with small $p_{T}$, is suitable for luminosity monitoring at ion colliders.

The layout of RHIC and LHC also makes it possible to detect neutrons close to $0^{\circ}$ from the incident beam direction. There is already interest in other measurments that could be performed with a zero degree neutron calorimeter.

\section{LUMINOSITY MEASUREMENT REQUIREMENTS}

The experimental program determines the requirements for luminosity precision at RHIC. The current goal for is to achieve an accuracy of $5-10 \%$ on the luminosity measurement for any given experiment but more stringent requirements could appear as the program evolves.

The required specification for luminosity precision is often stated in reference to measurements with different beam energies or species. This is particularly true in the measurement of 
departures from extrapolated pp behavior. There is apparently no alternative to measuring independently normalized production cross sections.

Another goal which is often stated for RHIC experiments is consistency in luminosity scale among different experiments- which would simplify comparison of results. This argues for the installation of identical luminosity measuring instruments that are independent of the rest of the experimental layout. This approach also has an enormous advantage in accelerator commissioning since the same trouble shooting tools can be used to tune each intersection region.

Finally, it should be recognized that instantaneous luminosity is also calculable from accelerator parameters $\left(I_{\text {beam }}, \sigma_{x}, \sigma_{y .}\right.$.) so long as beams are steered to give maximum overlap at the collision point. The Van der Meer method was employed at the first high energy light ion collider (the ISR) and should yield better than $10 \%$ precision at RHIC.

The purpose of the luminosity measurement using a standard physics cross section is to provide a real time monitor and to supply a cross check of the machine based determination. Since this exercise has been carried out many times for $\mathrm{pp}(\bar{p})$ colliders it is of interest to find a counterpart for the highest mass ion species.

A natural set of candidates is the virtual photo-nuclear processes since their cross sections grow rapidly with Atomic Number (approximately at least as fast as $Z^{4}$ ).

\section{CANDIDATE PROCESSES FOR LUMINOSITY MONITORING}

A number of processes may be considered for luminosity monitoring which all fail one of the following criteria:

- large cross section

- well understood acceptance for final state

- immunity from various possible backgrounds

Some examples, which illustrate this point follow. 


\section{A. Electron Pair Production Mediated by two Photon Exchange}

The electron pair production cross section [8] is very large when running with Au beams

$(\sigma \simeq 35 \mathrm{~kb})$ and this is a simple final state. However, because of the very steep $q^{2}$ dependence of the spectrum, the acceptance is difficult to calculate and may be very sensitive to details of the layout of the experiment- particularly magnetic fields. For example, in the STAR experiment most electrons are trapped by the detector's axial magnetic field before reaching the tracker. Because only the tail of a steeply falling spectrum is observed, the energy scale calibration also becomes part of the acceptance problem.

\section{B. Coulomb Dissociation with Neutron Emission}

This is also a large cross section with Au beam running $(\sigma=95 \mathrm{~b}$ [1] [2]) and it also gives rise to a simple final state- one or more neutrons which are emitted within a few mrads. of one of the beam directions. A calorimeter can be used to detect them and measure the number of neutrons by just dividing the total observed energy by the projectile energy per nucleon.

This process lacks a constraint on the point of production. Neutrons accompanying the outgoing beam could be produced by any number of "single-beam" processes, like beam-gas and beam- wall interactions. For this reason we reject this monitor in favor of a coincidence measurement.

\section{Nuclear Inelastic Cross Section}

Some sort of "minimum-bias" trigger will likely be in place for all experiments- generated by a coincidence of scintillator or Cerenkov hodoscopes on either side of the interaction region. This trigger will, by default, serve as the luminosity monitor for the different experiments. The acceptance of these hodoscopes is probably sensitive to other details of the experiment and not simply estimated from first principles. 
Typically the PHENIX "Beam Beam Counter" system at RHIC covers $3.1 \leq \eta \leq 4.0$ and a 2 arm coincidence is sensitive to $\geq 90 \%$ of the nuclear inelastic cross section in Au-Au collisions.

\section{CORRELATED FORWARD-BACKWARD COULOMB DISSOCIATION AT RHIC}

The Coulomb dissociation of a single beam nucleus at RHIC has already been considered in some detail. [1] [2] The cross section for heavy-ion dissociation may be accurately expressed in terms of (generally experimentally known) photo-dissociation cross sections of the same nucleus over an appropriate energy range of photon energies. This is the so-called Weizsacker-Williams expression

$$
\sigma_{d i s}=\frac{2 \alpha Z_{p}^{2}}{\pi \gamma^{2}} \int d \omega \omega \sigma_{p h}(\omega) \int_{b_{0}}^{\infty} b d b K_{1}^{2}\left(\frac{b \omega}{\gamma}\right) .
$$

We may define a probability of dissociation, $P(b)$ as a function of impact parameter $b$

$$
\sigma_{d i s}=2 \pi \int_{b_{0}}^{\infty} P(b) b d b
$$

Then inverting the order of integration in Eq. (1) we have

$$
P(b)=\frac{\alpha Z_{p}^{2}}{\pi^{2} \gamma^{2}} \int d \omega \omega \sigma_{p h}(\omega) K_{1}^{2}\left(\frac{b \omega}{\gamma}\right) .
$$

There is a problem with the analysis so far presented in that, as we will discover, this first order expression for $P(b)$ approaches unity near the grazing impact parameter for colliding heavy nuclei such as $\mathrm{Au}+\mathrm{Au}$. We will correct the expression to maintain unitarity later, but for the present we will continue with the first order analysis. The correlated dissociation cross section $\sigma_{c d}^{(1)}$ (here the (1) superscript indicates that this is a first order expression) requires both beam nuclei to be dissociated in an event. For identical colliding nuclei we have

$$
\sigma_{c d}^{(1)}=2 \pi \int_{b_{0}}^{\infty}[P(b)]^{2} b d b
$$




$$
\sigma_{c d}^{(1)}=\frac{2 \alpha^{2} Z^{4}}{\pi^{3} \gamma^{4}} \int_{b_{0}}^{\infty} b d b\left[\int d \omega \omega \sigma_{p h}(\omega) K_{1}^{2}\left(\frac{b \omega}{\gamma}\right)\right]^{2}
$$

The integral over $\dot{\omega}$ and $b$ can, of course, be evaluated numerically, and we will do so to obtain the numerical results we present below. However, it turns out that we can get a very accurate result analytically using the following scheme, which we will pursue for the present to elucidate the energy and impact parameter dependence of the mutual Coulomb dissociation. We evaluate the integral over $\omega$ in two pieces: above a certain value $\omega_{m}$ (say $18 \mathrm{GeV}) \sigma_{p h}^{(1)}$ is to a rough approximation a constant (which we call $\sigma_{c}$ ) thus simplifying its contribution as we show below; below $\omega_{m}$ we make the following approximationn.

$$
K_{1}^{2}\left(\frac{b \omega}{\gamma}\right)=\left(\frac{\gamma}{b \omega}\right)^{2}
$$

which is essentially exact (error of a part in a million) in the region of $\omega$ of the greatest contribution (the giant dipole resonance at about $14 \mathrm{MeV}$ ). Eq. (6) remains an excellent approximation up to $2 \mathrm{GeV}$, and introduces an error on the order of a few percent in the contribution between 2 and $18 \mathrm{GeV}$. We then have

$$
\sigma_{c d}^{(1)}=\frac{2 \alpha^{2} Z^{4}}{\pi^{3} \gamma^{4}} \int_{b_{0}}^{\infty} b d b\left[\int_{\omega_{0}}^{\omega_{m}} d \omega \omega \sigma_{p h}(\omega)\left(\frac{\gamma}{b \omega}\right)^{2}+\sigma_{c} \int_{\omega_{m}}^{\infty} d \omega \omega K_{1}^{2}\left(\frac{b \omega}{\gamma}\right)\right]^{2} .
$$

But in the higher $\omega$ region we make an error of less than a percent with the approximation

$$
\int_{\omega_{m}}^{\infty} d \omega \omega K_{1}^{2}\left(\frac{b \omega}{\gamma}\right) .=\left(\gamma^{2} / b^{2}\right) \ln \left(\frac{.681 \gamma}{b \omega_{m}}\right)
$$

We may now integrate over $b$ to obtain

$$
\sigma_{c d}^{(1)}=\frac{\alpha^{2} Z^{4}}{\pi^{3} b_{0}^{2}}\left\{\left[\int_{\omega_{0}}^{\omega_{m}} \frac{d \omega \sigma_{p h}(\omega)}{\omega}+\sigma_{c} \ln \left(\frac{.4131 \gamma}{b_{0} \omega_{m}}\right)\right]^{2}+\frac{\sigma_{c}^{2}}{4}\right\}
$$

It is clear that the evaluated cross section has a $1 / b_{0}^{2}$ dependence, where $b_{0}$ is the minimum impact parameter for which events are purely Coulomb, with no density overlap and no hadronic interactions.

At this point let us obtain an expression for the cross section that preserves unitarity in $P(b)$. If we assume independence of the various modes of dissociation, then the probability of 
at least one dissociation excitation of the nucleus is given by the usual Poisson distribution. Thus in our previous expression Eq.(4) we replace $[P(b)]^{2}$ with $\left[1-e^{-P(b)}\right]^{2}$. We ther evaluate the cross sections numerically on a computer.

Let us consider the standard design case of $100 \mathrm{GeV}+100 \mathrm{Gev} \mathrm{Au}+\mathrm{Au}$ at RHIC. Seen in the frame of the nucleus being dissociated, the equivalent $\gamma$ of the other ion providing the equivalent photons is 23,000 . The experimental photo-dissociation cross section $\sigma_{\mathrm{ph}}(\omega)$ that we utilzed is shown in Fig.(1), which we have taken from Ref. [2].

In Table I we present calculated first order and unitarity corrected mutual Coulomb dissociation cross sections for two values of $b_{0}, 15$ fermi and 17.3 fermi, corresponding to $r_{0}$ values of 1.29 and 1.49 respectively when one expresses $b_{0}$ in term of $2 \times r_{0}^{-} \mathrm{A}^{-1 / 3}$. Cross sections are tabulated as a function of a cutoff in $\omega$, which should correspond in some fashion to an experimental acceptance. For comparison, the corresponding cross sections for 2.76 $\mathrm{TeV}+2.76 \mathrm{TeV} \mathrm{Pb}+\mathrm{Pb}$ collisions at LHC are shown in Table II.

\section{EXPERIMENTAL CONSIDERATIONS}

Mutual dissociation could be measured with coincident detection of neutrons within a small angle of the incident beam direction. Neutrons which are emitted from the nucleus with kinetic energies of order $10 \mathrm{MeV}$ will be contained within a forward cone of opening angle less than 1 milliradian.

Measurements have been reported on the kinetic energy distributions from Heavy Ion single dissociation [4]. The kinetic energy of neutrons from both single emission $(1 \mathrm{n}, 1 \mathrm{p})$ and multiple emission (2p) were found to be less than $10 \mathrm{MeV}$.

\section{A. Forward $p_{t}$ Acceptance.}

Both the RHIC and LHC accelerator designs can accomodate so-called zero degree . calorimeters for neutron detection [5] [7]. In addition to energy measurement these detectors could provide precise time of flight information to aid in eliminating machine backgrounds. 
Transverse dimensions of possible detectors are limited by the distance between beam orbits in the regions where access for detector placement is possible.

In the case of RHIC, running at a nominal momentum of $100 \mathrm{Gev} / \mathrm{c} / \mathrm{u}$, and a detector size of $\pm 6 \mathrm{~cm}$. @ $20 \mathrm{~m}$ from the interaction point

$$
\begin{aligned}
p_{t}^{\max } & =\frac{0.06}{20} \times 100 \mathrm{GeV} / \mathrm{c} \\
& =300 \mathrm{MeV} / \mathrm{c}
\end{aligned}
$$

Therefore impacts from dissociation products should essentially always be contained.

At the LHC, with a nominal momentum of $2,760 \mathrm{GeV} / \mathrm{c} / \mathrm{u}$ and a detector size of \pm 4 $\mathrm{cm} @ 92 \mathrm{~m}$ from the interaction point

$$
\begin{aligned}
p_{t}^{\max } & =\frac{0.04}{92} \times 2,760 \mathrm{GeV} / \mathrm{c} \\
& =1,200 \mathrm{MeV} / \mathrm{c} .
\end{aligned}
$$

Thus in both cases there is no acceptance limitation- simply an instrumentation problem of measuring hadronic showers in such a small space.

\section{B. Semi-inclusive Cross section}

We note that photons contributing to the mutual dissociation process calculated here span a very wide range of energies. Although the nuclear final states will look very different above meson production threshold, we take the probability for emitting at least 1 neutron (with $\mathrm{T} \sim 10 \mathrm{MeV}$ ) to be a constant fraction of the total photonuclear cross section over the full energy range. This assumption implies some uncertainty since we know of no direct measuremnt of fragment Energy distributions in $\sim 10 \mathrm{GeV}$ photonuclear interactions. However measurements of "spectator nucleon" $P_{t}$ distributions [4] in nuclear interactions at AGS energies show that some neutron fragments will likely impact in the zero degree caloriemter region.

Similarly the cross section dependence on impact parameter cutoff, $b_{0}$, leads to an uncertainty in the dissociation cross section which also relates to possible detector bias. For 
example, one might be tempted to reject events where multiple particle production occurs in the central region. At impact parameters corresponding to

$$
b_{0}=2.0 \times A^{\frac{1}{3}} \times(1.5 f m .)
$$

or less we expect to see the onset of central particle production due to strong interactions.

A detailed study of particle production vs impact parameter should decide whether the fraction of the mutual dissociation cross section in which no central particle production occurs is more reliably calculable than the full cross section presented here. In this paper we treat the fully inclusive cross section corresponding to small angle leading neutron emission in each beam direction but without additional bias.

\section{Background from Uncorrelated Dissociation}

A potential source of background which cannot be distinguished from the process of interest is the accidental coincidence of single dissociation events in each beam. Since the beams have a bunch structure at both accelerators this accidental rate can be simply calculated given the bunch frequency and the single dissociation cross section.

Let us consider the case of RHIC at the design luminosity of $2 \times 10^{26} \mathrm{~cm}^{-2} \cdot \mathrm{s}^{-1}$ and the Day-1 bunch frequency of $4.8 \mathrm{Mhz}$. Then

$$
\begin{aligned}
R_{\text {sd }}=L \cdot \sigma & =0.92 \times 10^{-22} \times 2 . \times 10^{26} \\
& =1.8 \times 10^{4}
\end{aligned}
$$

is the rate of single dissociation events.

$$
R_{\text {crossing }}=4.8 \times 10^{6}
$$

is the rate of bunch crossings. A single dissociation event has a probability of $50 \% \times \frac{R_{\text {ad }}}{R_{\text {crossing }}}$ of overlapping another single dissociation event of the "other" beam. Since this is identical to the mutual correlated dissociation topology we can define 


$$
\sigma_{\text {acc }}=0.19 \times 10^{-2} \sigma_{s d}=0.17 \mathrm{barns}
$$

Clearly this effective cross section grows linearly with luminosity so long as the bunch frequency remains the same. The luminosity dependence could be used as a tool to evaluate this small background.

\section{CONCLUSION}

The mutual correlated Coulomb dissociation process of heavy ion beams at RHIC and the LHC has a large cross section, comparable to the total hadronic cross section. Because of its clean topology and large cross section, this process can be cleanly measured at RHIC and the LHC. The planned machine design for RHIC and LHC also permits the installation of relatively simple detectors which would have essentially $100 \%$ acceptance for this process.

It seems to us that these criteria make it suitable for luminosity monitoring in Heavy Ion running with the normal beams ( $\mathrm{Au}$ and $\mathrm{Pb}$ respectively) intended for RHIC and the LHC.

The luminosity monitor we have in- mind based on this process consists of recording . events in which one Beam Energy nucleon (100 GeV at RHIC) is emitted at within 1-2 milliradians in each of the beam directions. The cross section for this process at RHIC is computed to be about 3 barns. Nuclear interactions of the ion beams can also give rise to coincident forward neutrons. Since the nuclear interaction cross section is approximately 6 barns this gives rise to a potential background. We would propose a very rudimentary veto of nuclear interaction events requiring a small total multiplicity.

Nevertheless, as discussed above, there are some uncertainties in the borderline between the 2 classes of events. This uncertainty in the mutual dissociation cross section is estimated to be approximately $10 \%$ when expressed as an impact parameter and photon energy cutoff. This is illustrated in Table 1.

Since the cross section varies as $\mathrm{Z}^{4}$, this luminosity monitor would, however, lose its usefulnes for special runs with "light" ions such as Silicon and Sulfur. For light ion runs the 
experiments will have to complement the Van der Meer measurement of luminosity with a "minimum bias" based monitor based on the nuclear interaction rate. 


\section{REFERENCES}

[1] M. J. Rhoades-Brown and J. Weneser, BNL-47806.

[2] A. J. Baltz, M. J. Rhoades-Brown, and J. Weneser, BNL-63069.

[3] C.Augier et al. (UA4/2 Collaboration) CERN/PPE 93-115,1993.

[4] J.Barrette et al. Phys.Rev.C 51(1995) p.865.

[5] Calorimetry at 0 deg in RHIC, W.Christie, S.White,P.Gorodetzky and D.Lazic BNL62966, RHIC-Det-6.

[6] U.Amaldi et al., Phys. Lett. 62b, 460 (1976).

[7] Alice Technical Proposal, CERN/LHCC 95-71.

[8] J.Eichler and W.Meyerhof, Relativistic Atomic Collisions, Academic Press 1995, P.281. 


\section{FIGURES}

FIG. 1. Photonuclear dissociation cross sections utilized for the Au + Au Coulomb dissociation calculations. 


\section{TABLES}

TABLE I. First order $\sigma_{c d}^{1}$ and unitarity corrected $\sigma_{c d}$ (in barns) for Au $+A u$ at RHIC as a function of the cutoff photon energy $\omega_{\max }$ (in $\mathrm{MeV}$ ). Calculations are prsented for two values of the lower impact parameter cutoff (see text).

\begin{tabular}{|c|c|c|c|c|}
\hline \multirow[b]{2}{*}{$\omega_{\max }$} & \multicolumn{2}{|c|}{$\sigma_{c d}^{1}$} & \multicolumn{2}{|c|}{$\sigma_{c d}$} \\
\hline & $b_{0}=15$ & $b_{0}=17.32$ & $b_{0}=15$ & $b_{0}=17.32$ \\
\hline 25 & 1.65 & 1.23 & 1.31 & 1.04 \\
\hline 103 & 1.99 & 1.49 & 1.55 & 1.24 \\
\hline 440 & 3.10 & 2.32 & 2.29 & 1.84 \\
\hline 2000 & 4.21 & 3.16 & 2.98 & 2.42 \\
\hline 17840 & 5.12 & 3.84 & 3.50 & 2.86 \\
\hline$\infty$ & 5.91 & 4.39 & 3.90 & 3.19 \\
\hline
\end{tabular}

TABLE II. As in Table I, but for $\mathrm{Pb}+\mathrm{Pb}$ at LHC.

\begin{tabular}{|c|c|c|c|c|}
\hline \multirow[b]{2}{*}{$\omega_{\max }$} & \multicolumn{2}{|c|}{$\sigma_{c d}^{1}$} & \multicolumn{2}{|c|}{$\sigma_{c d}$} \\
\hline & $b_{0}=15$ & $\dot{b_{0}}=17.32$ & $b_{0}=15$ & $b_{0}=17.32$ \\
\hline 25 & 2.21 & 1.66 & 1.71 & 1.36 \\
\hline 103 & 2.66 & 2.00 & 2.01 & 1.61 \\
\hline 440 & 4.12 & 3.09 & 2.92 & 2.37 \\
\hline 2000 & 5.58 & 4.19 & 3.76 & 3.09 \\
\hline 17840 & 6.79 & 5.09 & 4.42 & 3.65 \\
\hline$\infty$ & 12.75 & 9.45 & 7.15 & 6.02 \\
\hline
\end{tabular}




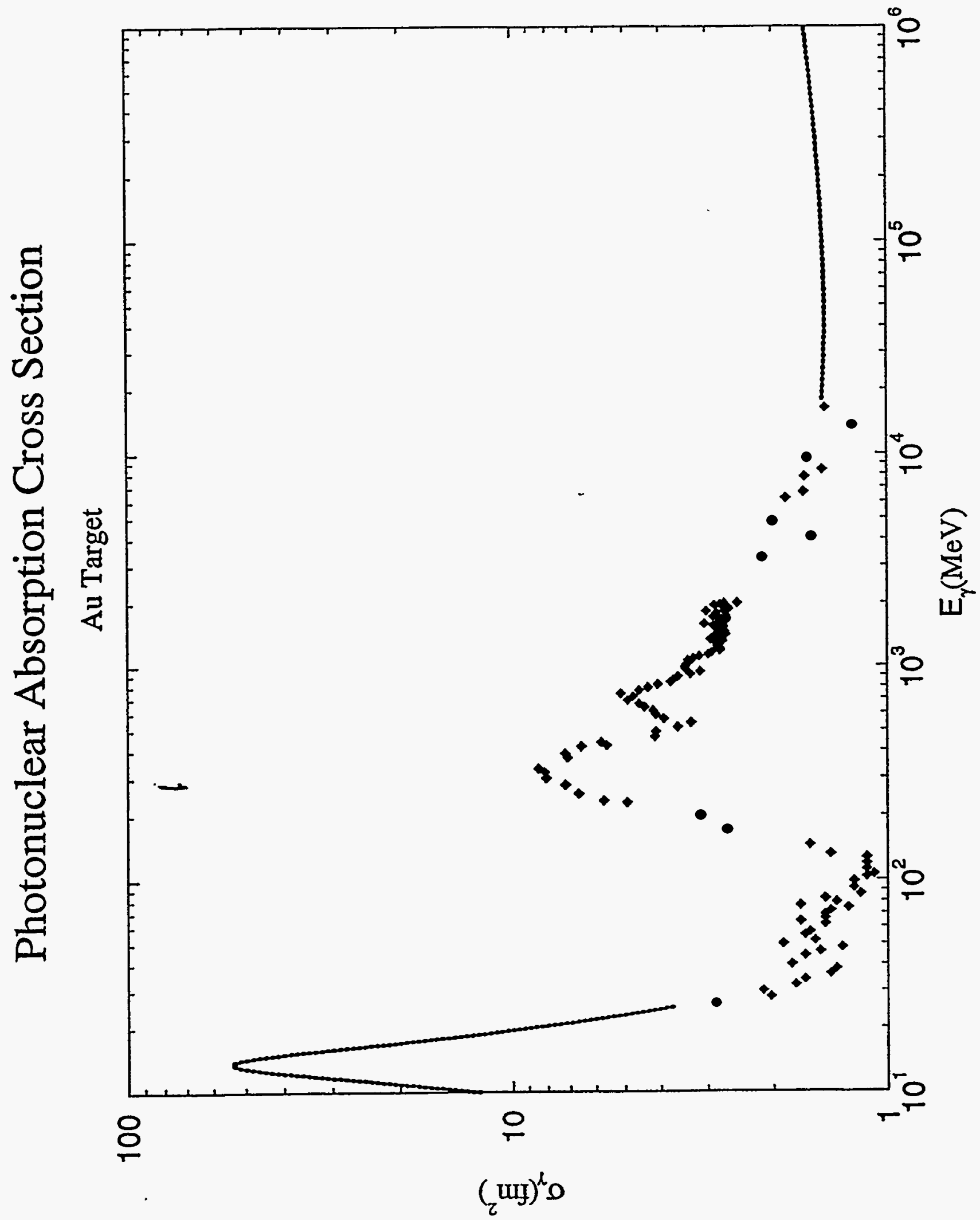

可 


\section{.}

